\title{
On the relationship between magnetic cloud field polarity and geoeffectiveness
}

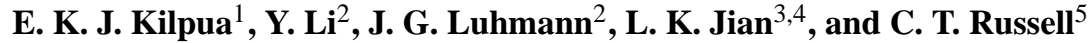 \\ ${ }^{1}$ Department of Physics, Division of geophysics and astronomy, P.O. Box 64, University of Helsinki, Finland \\ ${ }^{2}$ Space Sciences Laboratory, University of California, Berkeley, CA, USA \\ ${ }^{3}$ Department of Astronomy, University of Maryland, College Park, MD, USA \\ ${ }^{4}$ Heliophysics Science Division, NASA Goddard Space Flight Center, MD, USA \\ ${ }^{5}$ Institute of Geophysics and Planetary Physics, UCLA, Los Angeles, CA, USA
}

Correspondence to: E. K. J. Kilpua (emilia.kilpua@ helsinki.fi)

Received: 30 March 2012 - Revised: 1 June 2012 - Accepted: 6 June 2012 - Published: 13 July 2012

\begin{abstract}
In this paper, we have investigated geoeffectivity of near-Earth magnetic clouds during two periods concentrated around the last two solar minima. The studied magnetic clouds were categorised according to the behaviour of the Z-component of the interplanetary magnetic field $\left(B_{\mathrm{Z}}\right)$ into bipolar ( $B_{\mathrm{Z}}$ changes sign) and unipolar ( $B_{\mathrm{Z}}$ maintains its sign) clouds. The magnetic structure of bipolar clouds followed the solar cycle rule deduced from observations over three previous solar cycles, except during the early rising phase of cycle 24 when both $B_{Z}$ polarities were identified almost with the same frequency. We found a clear difference in the number of unipolar clouds whose axial field points south (S-type) between our two study periods. In particular, it seems that the lack of S-type unipolar clouds contributed to relatively low geomagnetic activity in the early rising phase of cycle 24 . We estimated the level of magnetospheric activity using a Dst prediction formula with the measured $B_{\mathrm{Z}}$ and by reversing the sign of $B_{\mathrm{Z}}$. We found that bipolar clouds with fields rotating south-to-north $(\mathrm{SN})$ and north-to-south (NS) were equally geoeffective, but their geoeffectiveness was clearly modified by the ambient solar wind structure. Geoeffectivity of NS-polarity clouds was enhanced when they were followed by a higher-speed solar wind, while the majority of geoeffective SN-polarity clouds lacked the trailing faster wind. A leading shock increased the geoeffectiveness of both NS- and SN-polarity clouds, in particular, in the case of an intense storm. We found that in 1995-1998, SN-polarity clouds were more geoeffective, while in 20062011 NS-polarity clouds produced more storms. A considerably larger fraction of events were trailed by a higher-
\end{abstract}

speed solar wind during our latter study period, which presumably increased geoeffectivity of NS-polarity. Thus, our study demonstrates that during low and moderate solar activity, geoeffectivity of opposite polarity bipolar clouds may depend significantly on the surrounding solar wind structure. In addition, different polarities also give different temporal storm evolutions: a storm from an SN-polarity cloud is expected to occur, on average, half-a-day earlier than a storm from an NS-polarity cloud.

Keywords. Solar physics, astrophysics, and astronomy (Flares and mass ejections)

\section{Introduction}

Interplanetary magnetic clouds are large-scale solar wind structures that are associated with coronal mass ejections (CMEs). Consequences of magnetic clouds are far-reaching in the heliosphere and in particular due to their intense and smoothly changing magnetic fields, magnetic clouds often drive magnetically disturbed periods in the Earth's magnetosphere (e.g., Webb et al., 2000; Richardson et al., 2001; Huttunen et al., 2005).

Magnetic clouds were first identified in the solar wind by Burlaga et al. (1981) as large-scale structures that exhibit smooth rotation of the magnetic field through a large angle with enhanced magnetic field and depressed proton temperature. Goldstein (1983) first proposed that magnetic clouds could be locally modelled as cylindrically symmetric flux tubes with force-free magnetic fields, fulfilling $\nabla \times \boldsymbol{B}=\alpha \boldsymbol{J}$, 
where $\boldsymbol{B}$ is the magnetic field and $\boldsymbol{J}$ the electric current density. A few years later, Burlaga (1988) noted that the magnetic field directional changes within magnetic clouds are presented to good approximation by a linear (constant $\alpha$ ) solution (Lundquist, 1950). The global configuration of a magnetic cloud is most commonly described in terms of a huge flux rope that is anchored to the Sun at both ends. This concept was originally based on the multi-spacecraft observations of a magnetic cloud by Burlaga et al. (1990).

Not all interplanetary CMEs (ICMEs) show magnetic cloud signatures. The plasma and magnetic field signatures of ICMEs vary greatly and only about $1 / 3$ of ICMEs observed close to the orbit of the Earth can be classified as magnetic clouds (Gosling, 1990). The study by Richardson and Cane (2004) showed that the fraction of magnetic clouds from all ICMEs varies with the solar activity cycle: close to solar minimum nearly all ICMEs are magnetic clouds, but the fraction decreases significantly when solar maximum is approached. On the other hand, as suggested by Jian et al. (2006) and Jian et al. (2008) using multiple ICME parameters, the absence of magnetic cloud signatures in many ICMEs may be a geometrical effect: their analysis demonstrated that in about two-thirds of the cases over a solar cycle, the spacecraft encounters the ICME so far from the centre that the central flux rope is not identifiable and this probability of encountering the central flux rope varies antiphase with solar activity.

Magnetic clouds are often divided into different categories based on how their magnetic field direction changes. Depending on whether the interplanetary magnetic field (IMF) north-south component $\left(B_{\mathrm{Z}}\right)$ changes the sign within a magnetic cloud, the cloud is designated as either bipolar or unipolar, respectively. This division reflects the tilt of the flux rope axis with respect to the ecliptic plane. Bipolar magnetic clouds have low inclination while unipolar clouds are oriented roughly perpendicular to the ecliptic plane. By labeling the direction of the magnetic field at the boundaries and at the centre of the ICME, Mulligan et al. (1998) presented eight flux rope categories: SEN, SWN, NES and NWS to present bipolar ICMEs, and WNE, ESW, ENW, and WSE to present unipolar ICMEs. For example, in the NWS type ICME the field rotates from the north $(\mathrm{N})$ at the front edge to the west $(\mathrm{W})$ at the centre and finally to the south (S) at the rear edge.

Studies from three previous solar cycles (Bothmer and Schwenn, 1998; Mulligan et al., 1998; Li and Luhmann, 2004; Huttunen et al., 2005; Li et al., 2011) have revealed that the polarity of $B_{Z}$ for bipolar clouds depends on the phase of the 22-year solar magnetic cycle. The polarity in magnetic clouds agrees with the global poloidal field of the Sun, except during the few years after the solar maximum when the polar field reverses. At that time, a mixture of SN- and NSpolarity clouds are observed and the dominant polarity of magnetic clouds does not reverse until the later part of the declining phase. From the late declining phase of odd (even) numbered solar cycles to the maximum of the next cycle NS (SN) clouds clearly dominate. This dependence on the solar magnetic cycle has been explained using rather simple pictures in which either filament fields (Bothmer and Schwenn, 1998) or large-scale solar fields (Mulligan et al., 1998) control magnetic cloud fields. However, studies covering over three solar cycles (Li et al., 2011) have shown that the relation between magnetic cloud fields observed in the solar wind and near the solar surface region is more complicated. The above listed studies have shown that the distribution to unipolar and bipolar clouds do not show an obvious solar cycle trend.

As dominance between SN- and NS-clouds changes with the solar magnetic cycle, it is important to know whether they lead to different geomagnetic consequences. The primary solar wind parameter that drives geomagnetic activity is the southward $B_{\mathrm{Z}}$, but high solar wind speed and density are known to increase geoeffectivity of solar wind structures (e.g. Pulkkinen et al., 2007, and references therein). The comparisons of geoeffectivity of different polarity magnetic clouds have yielded contradictory results: Zhang and Burlaga (1988) found that SN-clouds would cause larger magnetospheric storms than NS-clouds, while the study by $\mathrm{Li}$ and Luhmann (2004) concluded that they are equally geoeffective.

In the above described force-free flux rope geometry, the magnetic field magnitude peaks at the centre of the cloud and decreases to about half of this value at the boundaries. Thus, in principle, geoeffectivity of a magnetic cloud should not depend on its polarity as the maximum southward magnetic field value should be equal for SN and NS-clouds; only southward fields occur at the different parts of the clouds. However, the lateral expansion of the cloud and its interaction with the ambient solar wind may affect the magnetic field, speed and density profiles of the cloud and consequently modify its geoeffectiveness.

Fenrich and Luhmann (1998) proposed that NS-clouds might cause larger geomagnetic storms when they are compressed from behind by a fast solar wind stream. The authors found that trailing fast streams were observed for about $40-45 \%$ of bipolar clouds, thus, making this compression a likely scenario. A few case studies were presented where the geoeffectivity of an NS-cloud was enhanced by a trailing stream, but no statistical results were presented on which type produced a stronger storm. In addition, it has been speculated (e.g. Zhang and Burlaga, 1988) that fast SN-clouds would be more geoeffective because sheath fields often initiate the storm and, thus, may strengthen the disturbance caused by the closely following southward fields in the leading portion of the cloud.

In turn, S-type unipolar magnetic clouds are expected to be particularly geoeffective as for them (in the idealized picture) the field is purely southward at the centre where magnetic field magnitude peaks. In the study by Huttunen et al. (2005) covering the years 1997-2003, all 15 identified S-type 
magnetic clouds caused a storm and nine of them were intense magnetic storms.

In this paper, we have investigated geomagnetic consequences of bipolar and unipolar magnetic clouds identified during two periods concentrated around the last two solar minima. During our first study period, SN-clouds prevailed, while during the second period, NS-clouds were expected to dominate. These two periods are also interesting to compare as they were associated with clearly different solar minimum conditions. It is well known that the late declining phase of solar cycle 23 and the following minimum were unusually long and deep when compared to three previous corresponding phases (e.g., Russell et al., 2010; Jian et al., 2011). The global solar magnetic field had significant differences between our study periods that were reflected throughout the heliosphere (e.g., Lee et al., 2009; Cremades et al., 2011; Jian et al., 2011) and as we will show, affected the solar wind structure surrounding magnetic clouds. The minimum following cycle 22 was a "typical" solar minimum featuring a dipole-like solar magnetic field and large polar coronal holes, while during the recent minimum, the global magnetic field of the Sun had a multipole structure, and as a consequence, low- and mid-latitude coronal holes were frequently present (Abramenko et al., 2010). The properties of magnetic clouds vary considerably from event to event and, thus, it is difficult to estimate whether the geoeffectiveness of bipolar clouds depends on its polarity. As discussed above, previous studies have yielded contradictory results on the importance of cloud's polarity for its geoeffectiveness. To overcome this problem, we examine the geomagnetic response of each cloud with original and reversed polarity, obtained by reversing the sign of $B_{\mathrm{Z}}$ and using an empirical Dst prediction formula (see Sect. 2 for details). This approach allows us to distinguish how much for a given cloud its polarity and ambient solar wind structure affected its geoeffectivity. In addition, using both original and reversed polarity improves our statistics by doubling the number of events for which we can estimate the geomagnetic response. Furthermore, we have studied magnetospheric storms driven by unipolar clouds.

\section{Data and definitions}

Our first study interval (Period 1) lasted four years, from 1995 through 1998, and the second interval (Period 2) extended six years, from 2006 through 2011 (see Fig. 1). This selection was based on the availability of continuous solar wind plasma and magnetic field measurements by the nearEarth spacecraft, the dominance of opposite polarity bipolar magnetic clouds (see Sect. 1), and the levels of sunspot activity (see Fig. 1). As indicated by the monthly sunspot number shown in Fig. 1, the investigated periods include the late declining phases of cycles 22 and 23, following solar minima, and the early rising phases of cycles 23 and 24. Note that Period 2 is longer than Period 1 by two years because, as dis-

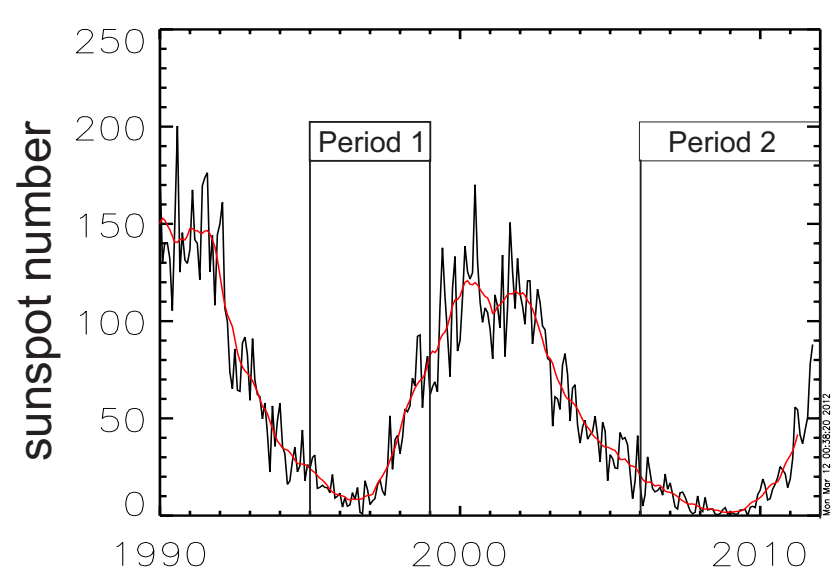

Fig. 1. Monthly (black) and monthly smoothed (red) sunspot numbers from Solar Influences Data Center from January 1990 through December 2011. Our study periods are indicated in the figure.

cussed in introduction, the low solar activity period following cycle 23 was unusually long and deep. It is seen from Fig. 1 that the sunspot numbers were roughly at similar levels during 1995 and 2006, as well as during 1998 and 2011. Solar cycle 23 peaked in March 2000, with the maximum (monthly smoothed) sunspot number 120.8 and according to current predictions (by Hathaway/NASA/MSFC), cycle 24 will peak in early 2013 with the maximum sunspot number 59.

We have selected our magnetic clouds using online catalogs (Wind magnetic cloud list at http://wind.nasa.gov/mfi/ mag_cloud_pub1.html, UCLA ICME list at http://www.srl. caltech.edu/ACE/ASC/DATA/level3/) and published magnetic cloud lists (Huttunen et al., 2005; Li et al., 2011). In addition, we have surveyed in situ observations of the IMF and solar wind plasma parameters from the Near-Earth Heliospheric database (OMNI). OMNI is composed from measurements by the near-Earth spacecraft and the data has been shifted to the magnetopause. We have required that our events have an enhanced magnetic field magnitude with peak value over $10 \mathrm{nT}$, depressed proton temperature and plasma beta as well as low variance magnetic field with smoothly rotation magnetic field vector over a time interval on the order of a day, consistent with the original definition by Burlaga et al. (1981).

Following the categorisation by Bothmer and Schwenn (1998) and by Mulligan et al. (1998) we divided our events into bipolar and unipolar clouds, and further to NS, SN, S and $\mathrm{N}$-types emphasising the behaviour of $B_{\mathrm{Z}}$. We have not indicated the direction of the axial field for bipolar clouds nor the direction of the poloidal field at the cloud's boundaries for unipolar clouds because, in this paper, we focus on their geoeffectivity that primarily depends on the characteristics of $B_{Z}$. The magnetic clouds, selected in this study, are listed in Tables 1 and 2.

We have studied geomagnetic effects of magnetic clouds using a Dst prediction formula presented originally by 
Burton et al. (1975). Dst is a 1-h geomagnetic activity index that was developed to measure the strength of the equatorial ring current. It is derived using measurements from four low-latitude magnetometer stations distributed roughly evenly around the Earth. In this paper, we decided to use estimated Dst because at the time of this study final Dst values were not available for all investigated years and because our purpose was to investigate geomagnetic consequences of magnetic clouds with both original and reversed $B_{\mathrm{Z}}$. According to Burton et al. (1975) Dst can be predicted using the following formula:

$$
\begin{aligned}
\frac{d\left(\text { Dst }^{*}\right)}{d t} & =F(E)-a \mathrm{Dst}^{*}, \text { where } \\
\mathrm{Dst}^{*} & =\mathrm{Dst}-b \sqrt{P_{\mathrm{dyn}}}+c, \\
b & =0.20 \mathrm{nT} \sqrt{\mathrm{eV} \mathrm{cm}^{3}} \text { and } \\
c & =20 \mathrm{nT} .
\end{aligned}
$$

Dst* is a pressure corrected Dst, presenting the disturbance ring current, where the contribution from magnetopause currents and the quiet time ring current have been removed. $P_{\mathrm{dyn}}$ is the solar wind dynamic pressure and the values for constants $b$ and $c$ given here were derived by O'Brien and McPherron (2000) using 30 years of hourly Dst and solar wind data. $F(E)$ is the ring current injection rate and we have used the form that depends on $P_{\mathrm{dyn}}$ and solar wind dawn-dusk electric field $E_{\mathrm{Y}}$ (estimated here as a product of $\mathrm{X}$-component of the solar wind speed and $B_{\mathrm{Z}}$ ):

$$
\begin{aligned}
F(E) & =d P_{\mathrm{dyn}}^{1 / 3}\left(E_{\mathrm{Y}}-0.5\right) \\
d & =-1.2 \times 10^{-3} \mathrm{nT}\left(\mathrm{smV} \mathrm{m}^{-1}\right)^{-1} .
\end{aligned}
$$

This injection rate was used by Fenrich and Luhmann (1998), and its functional form was based on the study by Murayama (1982).

Figure 2 shows an example of a bipolar magnetic cloud that was detected 10-11 January 1997. Figure $2 f$ shows the measured $B_{\mathrm{Z}}$ that rotates smoothly from south to north during the passage of the cloud. When the sign of $B_{Z}$ is reversed (Fig. 2h), the magnetic field rotates in the opposite way, from north to south. In the former case, we define that the cloud is an original-SN cloud, and in the latter case, where the sign of $B_{\mathrm{Z}}$ was reversed, it is called a reverse- $S N$ cloud. Using this terminology, original-SN and reversed-NS clouds have SN-polarity, and original-NS and reversed-SN clouds have NS-polarity. Dst values predicted using the above described modified Burton's formula with original $B_{\mathrm{Z}}$ are given in Fig. $2 \mathrm{~g}$ and with reversed $B_{Z}$ in Fig. 2i. The Dst minimum for the original-SN cloud was $-90 \mathrm{nT}$, and slightly stronger, $-110 \mathrm{nT}$ for the reverse-SN cloud.

We defined a magnetic cloud to be followed by increased solar wind speed if the solar wind speed averaged over a 24-h period after the cloud's trailing edge was at least $50 \mathrm{~km} \mathrm{~s}^{-1}$ larger than the average solar wind speed in the latter half of the cloud. For the magnetic cloud shown in Fig. 2 the average solar wind speed in the latter half of the cloud was

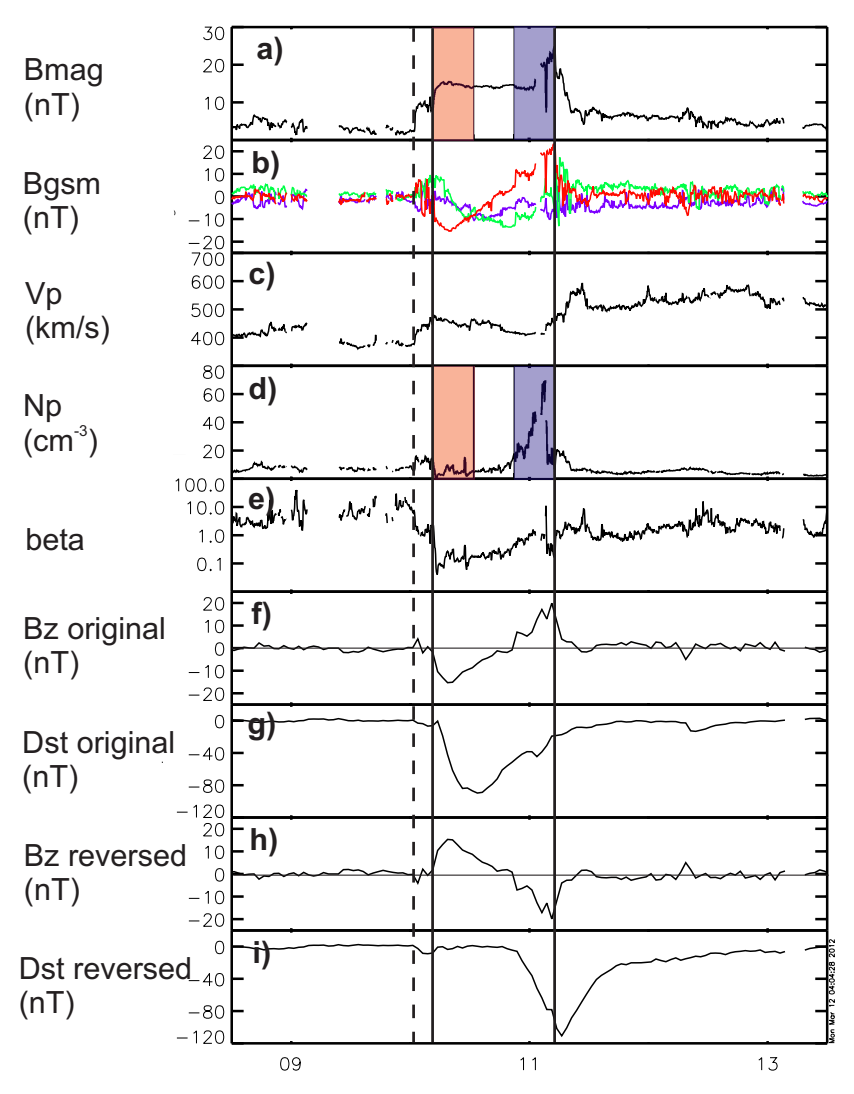

Fig. 2. An example of a bipolar SN-type magnetic cloud detected on 10-11 January 1997. The solar wind plasma and magnetic field measurements are from the OMNI-data base. The panels from top to bottom give: (a) magnetic field magnitude, (b) magnetic field components in GSM-coordinate system (purple: $B_{X}$, green: $B_{Y}$, red: $B_{\mathrm{Z}}$, (c) solar wind speed, (d) density, and (e) plasma beta. Panels (f) and (h) give the 1-h measured and reversed $B_{Z}$, respectively, and panels (g) and (i) Dst predicted from the modified Burton et al. (1975) formula (see text) using the measured and reversed $B_{Z}$, respectively. The magnetic cloud interval is bounded between a pair of solid lines and the interplanetary shock is indicated by a dashed line. In panels (a) and (d) red-shaded (blue-shaded) regions indicate the intervals that were used to calculated leading (trailing) magnetic field magnitude and density.

$426 \mathrm{~km} \mathrm{~s}^{-1}$, and the average solar wind speed $24 \mathrm{~h}$ after the cloud's trailing edge $520 \mathrm{~km} \mathrm{~s}^{-1}$, yielding the difference of $94 \mathrm{~km} \mathrm{~s}^{-1}$. Since the trailing high-speed stream may compress the end part of the cloud, we calculated the ratio of the magnetic field magnitude and density in the leading and trailing parts of the cloud. The leading magnetic field $\left(B_{\mathrm{LE}}\right)$ and density $\left(N_{\mathrm{LE}}\right)$ were calculated as averages during the first third of the cloud (red-shaded regions in Fig. 2a and d) and the trailing magnetic field $\left(B_{\mathrm{TE}}\right)$ and density $\left(N_{\mathrm{TE}}\right)$ as averages during the last third of the cloud (blue-shaded regions in Fig. $2 \mathrm{a}$ and d). If $B_{\mathrm{TE}} / B_{\mathrm{LE}}$ was over 1.1, we defined that cloud's trailing fields as compressed, and if $N_{\mathrm{TE}} / N_{\mathrm{LE}}$ was over 1.5 , we termed that there was a trailing density enhancement. For the January 1997 cloud, these ratios were 1.17 
Table 1. Magnetic clouds during 1995-1998 (Period 1). The first three columns give the times when the shock and the magnetic cloud leading and trailing edges arrived at the magnetopause. Column "type" indicates the original $B_{\mathrm{Z}}$ type of the magnetic cloud. The next two columns give the minimum Dst value (if $<-50 \mathrm{nT}$ ) and the time when it was observed as estimated from the modified Burton's formula (see Sect. 2) for measured and reversed $B_{Z}$. Note that the estimated Dst values may differ from the Dst values calculated from magnetometer recordings. The last column indicates by " $\mathrm{H}$ " if the magnetic cloud was associated with a trailing higher-speed solar wind (HSS) and by "I" if it was overtaken by another ICME.

\begin{tabular}{|c|c|c|c|c|c|c|}
\hline Shock (UT) & Cloud, start (UT) & Cloud, stop (UT) & Type & Original Dst & Reversed Dst & HSS/ICME \\
\hline \multicolumn{7}{|l|}{1995} \\
\hline - & $2 / 8,04: 20$ & $2 / 8,22: 25$ & SN & $-69 \mathrm{nT} 2 / 8,10: 00 \mathrm{UT}$ & _- & - \\
\hline $3 / 4,01: 10$ & $3 / 4,12: 00$ & $3 / 5,03: 35$ & $\mathrm{~S}$ & $-71 \mathrm{nT} 3 / 5,00: 00 \mathrm{UT}$ & - & - \\
\hline - & $4 / 3,01: 00$ & $4 / 4,12: 30$ & NS & - & $-54 \mathrm{nT} 4 / 3,18: 00 \mathrm{UT}$ & _- \\
\hline - & $5 / 13,11: 30$ & $5 / 13,17: 50$ & $\mathrm{~N}$ & - & $-54 \mathrm{nT} 5 / 13,17: 00 \mathrm{UT}$ & - \\
\hline - & $6 / 30,15: 50$ & $7 / 2,11: 30$ & SN & $-59 \mathrm{nT} 6 / 30,22: 00 \mathrm{UT}$ & - & _- \\
\hline $8 / 22,12: 57$ & $8 / 22,19: 27$ & $8 / 23,21: 00$ & SN & $-57 \mathrm{nT}$ & - & - \\
\hline- & $9 / 27,13: 15$ & $9 / 27,21: 55$ & $\mathrm{~S}$ & $-89 \mathrm{nT}$ 9/27, 21:00 UT & _- & _- \\
\hline $10 / 18,11: 29$ & $10 / 18,19: 50$ & $10 / 19,01: 20$ & SN & -114 nT 10/19, 05:00 UT & $-167 \mathrm{nT} 10 / 20,02: 00 \mathrm{UT}$ & $\mathrm{H}$ \\
\hline $12 / 15,04: 37$ & $12 / 16,01: 10$ & $12 / 16,19: 00$ & $\mathrm{SN}$ & $-54 \mathrm{nT} 12 / 16,17: 00 \mathrm{UT}$ & - & - \\
\hline \multicolumn{7}{|c|}{1996} \\
\hline - & $5 / 27,14: 34$ & $5 / 29,11: 20$ & SN & - & $-91 \mathrm{nT} \mathrm{5/29,09:00 \textrm {UT }}$ & $\mathrm{H}$ \\
\hline $7 / 1,13: 55$ & $7 / 1,22: 10$ & $7 / 2,09: 50$ & $\mathrm{SN}$ & _- & - & $\mathrm{H}$ \\
\hline - & $12 / 24,03: 30$ & $12 / 25,12: 10$ & NS & - & $-59 \mathrm{nT} 12 / 24,14: 00 \mathrm{UT}$ & - \\
\hline \multicolumn{7}{|l|}{1997} \\
\hline $1 / 10,00: 50$ & $1 / 10,04: 30$ & $1 / 11,05 / 20$ & SN & $-90 \mathrm{nT} 1 / 10,13: 00 \mathrm{UT}$ & $-110 \mathrm{nT} 1 / 11,06: 00 \mathrm{UT}$ & $\mathrm{H}$ \\
\hline $2 / 9,13: 05$ & $2 / 10,03: 25$ & $2 / 10,19: 15$ & $\mathrm{~S}$ & $-71 \mathrm{nT} 2 / 10,19: 00 \mathrm{UT}$ & - & - \\
\hline- & $4 / 11,06: 20$ & $4 / 11,20: 35$ & $\mathrm{~N}$ & - & $-155 \mathrm{nT} 4 / 11,16: 00 \mathrm{UT}$ & - \\
\hline - & $4 / 21,12: 00$ & $4 / 23,04: 00$ & SN & $-69 \mathrm{nT} 4 / 21,23: 00 \mathrm{UT}$ & - & $\mathrm{H}$ \\
\hline $5 / 15,01: 20$ & $5 / 15,09: 00$ & $5 / 15,23: 25$ & SN & $-136 \mathrm{nT} 5 / 15,14: 00 \mathrm{UT}$ & $-61 \mathrm{nT} 5 / 16,03: 00 \mathrm{UT}$ & - \\
\hline - & $6 / 9,05: 55$ & $6 / 9,23: 02$ & SN & -73 nT $6 / 9,10: 00$ UT & - & - \\
\hline- & $7 / 15,09: 05$ & $7 / 16,07: 16$ & $\mathrm{SN}$ & $-63 \mathrm{nT} 7 / 15,19: 00 \mathrm{UT}$ & - & - \\
\hline - & $8 / 3,14: 25$ & 8/4, 02:01 & SN & $-74 \mathrm{nT} 8 / 30,19: 00 \mathrm{UT}$ & - & - \\
\hline- & $9 / 3,13: 20$ & $9 / 3,22: 30$ & NS & $-60 \mathrm{nT} \mathrm{9/3,21:00} \mathrm{UT}$ & - & $\mathrm{H}$ \\
\hline - & 9/21, 22:00 & $9 / 22,17: 15$ & $\mathrm{~N}$ & - & $-74 \mathrm{nT}$ 9/22, 18:00 UT & - \\
\hline $10 / 1,17: 10$ & $10 / 1,16: 20$ & $10 / 2,22: 40$ & $\mathrm{~N}$ & - & $-92 \mathrm{nT} \mathrm{10/3,00:00 \textrm {UT }}$ & - \\
\hline - & $10 / 10,21: 00$ & $10 / 12,02: 30$ & SN & $-74 \mathrm{nT} 10 / 11,04: 00 \mathrm{UT}$ & $-59 \mathrm{nT} \mathrm{10/12,00:00 \textrm {UT }}$ & - \\
\hline $11 / 6,22: 20$ & $11 / 7,06: 30$ & $11 / 8,14: 30$ & SN & $-72 \mathrm{nT} 11 / 7,10: 00 \mathrm{UT}$ & $-90 \mathrm{nT} 11 / 8,05: 00 \mathrm{UT}$ & - \\
\hline \multirow{2}{*}{\multicolumn{7}{|c|}{$\begin{array}{l}11 / 22,09.5 J \quad 11 / 2 L, 19.1 J \\
1998\end{array}$}} \\
\hline & & & & & & \\
\hline $1 / 6,14: 50$ & $1 / 7,03: 00$ & 1/8, 09:00 & $\mathrm{N}$ & - & $-82 \mathrm{nT} 1 / 8,09: 00 \mathrm{UT}$ & - \\
\hline $1 / 28,16: 25$ & $1 / 29,13: 00$ & $1 / 31,00: 25$ & $\mathrm{~S}$ & $-52 \mathrm{nT} 1 / 30,12: 00 \mathrm{UT}$ & - & - \\
\hline - & $2 / 4,06: 00$ & $2 / 5,23: 00$ & SN & - & - & - \\
\hline - & $2 / 17,10: 50$ & $2 / 18,15: 50$ & $\mathrm{~S}$ & $-101 \mathrm{nT} 2 / 18,03: 00 \mathrm{UT}$ & _- & $\mathrm{H}$ \\
\hline $2 / 18,08: 30$ & $2 / 18,21: 50$ & $2 / 20,01: 10$ & NS & - & $-51 \mathrm{nT} \mathrm{2/19,06:00 \textrm {UT }}$ & - \\
\hline $3 / 4,12: 00$ & $3 / 4,15: 40$ & $3 / 6,03: 30$ & $\mathrm{SN}$ & - & $-74 \mathrm{nT} 3 / 5,22: 00 \mathrm{UT}$ & - \\
\hline $5 / 1,21: 50$ & $5 / 2,09 ; 45$ & $5 / 3,17: 50$ & $\mathrm{~S}$ & $-1045 / 2,18: 00 \mathrm{UT}$ & - & $\mathrm{H}$ \\
\hline $5 / 15,14: 50$ & $5 / 15,23: 30$ & $5 / 16,09: 05$ & $\mathrm{~N}$ & - & $-72 \mathrm{nT} 5 / 16,15: 00 \mathrm{UT}$ & $\mathrm{H}$ \\
\hline - & $6 / 2,11: 18$ & $6 / 2,20: 40$ & SN & - & - & - \\
\hline - & 6/14, 05:00 & $6 / 14,23: 30$ & SN & - & - & - \\
\hline - & $6 / 24,14: 00$ & $6 / 25,22: 20$ & SN & _- & $-112 \mathrm{nT} 6 / 25,22: 00 \mathrm{UT}$ & $\mathrm{H}, \mathrm{I}$ \\
\hline $8 / 19,19: 00$ & $8 / 20,10: 20$ & $8 / 21,20: 50$ & SN & $-66 \mathrm{nT} 20 / 20,19: 00 \mathrm{UT}$ & $-60 \mathrm{nT} 8 / 21,13: 00 \mathrm{UT}$ & - \\
\hline $10 / 18,20: 10$ & $10 / 19,04: 40$ & $10 / 20,07: 30$ & $\mathrm{~S}$ & $-129 \mathrm{nT} 10 / 19,15: 00 \mathrm{UT}$ & - & $\mathrm{H}$ \\
\hline $11 / 7,08: 20$ & $11 / 7,22: 20$ & $11 / 8,17: 40$ & SN & $-143 \mathrm{nT} 11 / 8,07: 00 \mathrm{UT}$ & $-217 \mathrm{nT} 11 / 8,20: 00 \mathrm{UT}$ & I \\
\hline $11 / 8,04: 40$ & $11 / 8,23: 50$ & $11 / 10,02: 20$ & $\mathrm{~S}$ & $-116 \mathrm{nT} 11 / 9,11: 00 \mathrm{UT}$ & - & - \\
\hline - & $11 / 13,04: 20$ & $11 / 14,08: 00$ & $\mathrm{~S}$ & $-115 \mathrm{nT} 11 / 13,19: 00 \mathrm{UT}$ & - & - \\
\hline
\end{tabular}


Table 2. Magnetic clouds during 2006-2011 (Period 2). The columns are the same as in Table 1.

\begin{tabular}{|c|c|c|c|c|c|c|}
\hline Shock (UT) & Cloud, start (UT) & Cloud, stop (UT) & Type & Original Dst & Reversed Dst & HSS/ICME \\
\hline \multicolumn{7}{|l|}{2006} \\
\hline - & $1 / 25,12: 35$ & $1 / 26,03: 25$ & NS & - & - & $\mathrm{H}$ \\
\hline - & $2 / 5,19: 00$ & $2 / 6,12: 45$ & NS & _- & _- & - \\
\hline $4 / 13,11: 12$ & $4 / 13,15: 45$ & $4 / 14,11: 25$ & NS & $-119 \mathrm{nT} 4 / 14,11: 00 \mathrm{UT}$ & $-97 \mathrm{nT} 4 / 13,21: 00 \mathrm{UT}$ & - \\
\hline - & $5 / 3,20: 45$ & $5 / 4,14: 35$ & NS & $-55 \mathrm{nT} 5 / 4,14: 00 \mathrm{UT}$ & - & _- \\
\hline - & 9/30, 09:04 & $9 / 30,23: 00$ & NS & - & $-75 \mathrm{nT}$ 9/30, 16:00 UT & $\mathrm{H}$ \\
\hline - & $11 / 29,06: 05$ & $11 / 30,14: 20$ & NS & $-67 \mathrm{nT} 11 / 30,14: 00 \mathrm{UT}$ & $-75 \mathrm{nT} 11 / 29,18: 00 \mathrm{UT}$ & - \\
\hline $\begin{array}{l}12 / 14,13: 52 \\
2007\end{array}$ & \multicolumn{5}{|c|}{2007} & - \\
\hline - & $1 / 14,12: 35$ & $1 / 15,08: 25$ & NS & _- & _- & _- \\
\hline - & $5 / 21,23: 15$ & $5 / 22,14: 20$ & $\mathrm{~N}$ & - & $-71 \mathrm{nT} 5 / 22,09: 00 \mathrm{UT}$ & - \\
\hline \multicolumn{7}{|c|}{ 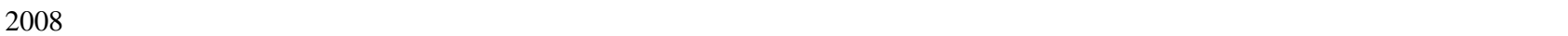 } \\
\hline $\begin{array}{l}- \\
2009\end{array}$ & \multicolumn{5}{|r|}{ 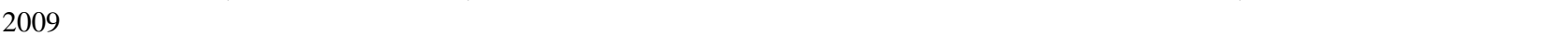 } & $\mathrm{H}$ \\
\hline $2 / 3,19: 21$ & $2 / 4,00: 55$ & $2 / 4,18: 20$ & NS & $-52 \mathrm{nT} 2 / 4,18: 00 \mathrm{UT}$ & - & - \\
\hline - & $3 / 12,02: 40$ & $3 / 13,02: 00$ & $\mathrm{~N}$ & - & $-81 \mathrm{nT} 3 / 12,20: 00 \mathrm{UT}$ & $\mathrm{H}$ \\
\hline - & $7 / 21,04: 20$ & $7 / 22,06: 10$ & NS & $-67 \mathrm{nT} 7 / 22,05: 00 \mathrm{UT}$ & - & $\mathrm{H}$ \\
\hline - & $8 / 5,12: 20$ & $8 / 6,06: 30$ & $\mathrm{~N}$ & _- & $-82 \mathrm{nT} 8 / 6,02: 00 \mathrm{UT}$ & $\mathrm{H}$ \\
\hline - & $10 / 29,06: 50$ & $10 / 30,00: 35$ & NS & - & - & - \\
\hline \multicolumn{7}{|l|}{2010} \\
\hline - & $2 / 7,23: 55$ & $2 / 8,23: 24$ & $\mathrm{~N}$ & - & - & - \\
\hline - & $5 / 18,05: 55$ & $5 / 19,10: 00$ & SN & - & $-64 \mathrm{nT} 5 / 19,19: 00 \mathrm{UT}$ & $\mathrm{H}$ \\
\hline $5 / 28,02: 45$ & $5 / 28,20: 00$ & $5 / 29,21: 30$ & $\mathrm{~S}$ & $-84 \mathrm{nT} 5 / 29,15: 00 \mathrm{UT}$ & - & $\mathrm{H}, \mathrm{I}$ \\
\hline $8 / 3,18: 10$ & $8 / 4,03: 15$ & $8 / 4,08: 20$ & SN & - & $-80 \mathrm{nT} 8 / 4,08: 00 \mathrm{UT}$ & $\mathrm{H}$ \\
\hline- & $8 / 4,11: 25$ & $8 / 5,01: 35$ & NS & $-58 \mathrm{nT} 8 / 5,01: 00 \mathrm{UT}$ & - & - \\
\hline \multicolumn{7}{|l|}{2011} \\
\hline $2 / 4,01: 49$ & $2 / 4,14: 10$ & $2 / 4,21: 00$ & NS & $-61 \mathrm{nT} 2 / 5,01: 00 \mathrm{UT}$ & - & $\mathrm{H}$ \\
\hline $2 / 14,14: 45$ & $2 / 14,20: 20$ & $2 / 15,02: 30$ & SN & - & $-62 \mathrm{nT} 2 / 15,04: 00 \mathrm{UT}$ & $\mathrm{H}$ \\
\hline $3 / 29,15: 50$ & $3 / 30,01: 00$ & $3 / 31,18: 00$ & $\mathrm{~N}$ & - & $-87 \mathrm{nT} 3 / 30,20: 00 \mathrm{UT}$ & - \\
\hline - & $5 / 28,06: 10$ & $5 / 28,21: 45$ & SN & $-78 \mathrm{nT} 5 / 28,13: 00 \mathrm{UT}$ & $-54 \mathrm{nT} 5 / 28,21: 00 \mathrm{UT}$ & $\mathrm{H}$ \\
\hline $6 / 4,20: 45$ & $6 / 5,01: 35$ & $6 / 5,09: 10$ & $\mathrm{~N}$ & _- & $-130 \mathrm{nT} 6 / 5,08: 00 \mathrm{UT}$ & - \\
\hline $10 / 24,18: 25$ & $10 / 25,01: 20$ & $10 / 25,15: 40$ & $\mathrm{~N}$ & _- & $-110 \mathrm{nT} 10 / 25,15: 00 \mathrm{UT}$ & - \\
\hline
\end{tabular}

Table 3. Percentages of magnetic clouds during our two study periods associated with a higher-speed trailing solar wind (HSS) and trailing density and magnetic field enhancements. For definitions see Sect. 2.

\begin{tabular}{lccc}
\hline Study period & HSS & $N_{\mathrm{TE}} / N_{\mathrm{LE}}>1.5$ & $B_{\mathrm{TE}} / B_{\mathrm{LE}}>1.1$ \\
\hline $1995-1998$ & $26 \%$ & $26 \%$ & $31 \%$ \\
$2006-2011$ & $48 \%$ & $67 \%$ & $45 \%$ \\
\hline
\end{tabular}

and 4.84, respectively, thus indicating a compression of the end part.

Using the criteria defined above, $35 \%$ of our magnetic clouds were trailed by higher-speed solar wind. $64 \%$ of the events overtaken by faster solar wind were associated with compressed trailing magnetic fields, and $72 \%$ with compressed trailing densities. For comparison, only $20 \%$ and
$25 \%$ of clouds that were followed by slow or declining fast solar wind had increased magnetic fields and densities in the end part of the cloud, respectively. Our dataset includes three magnetic clouds that were interacting with another ICME. This ICME-ICME interaction also resulted in a significant compression of the tail part of the preceding cloud as well as for two events to increasing solar wind speed after the cloud's trailing edge.

Table 3 separately shows, for our study periods, the fraction of clouds that were associated with faster trailing solar wind and magnetic field and density enhancements in the cloud's tail part. It is seen that a considerably larger fraction of clouds interacted with the following higher-speed solar wind stream in 2006-2011 than in 1995-1998: during Period $1,26 \%$ of clouds were associated with increased solar wind after the cloud's trailing edge, while this was the case for $48 \%$ of events during Period 2. Consequently, as seen from Table 3, the fraction of events associated with trailing 

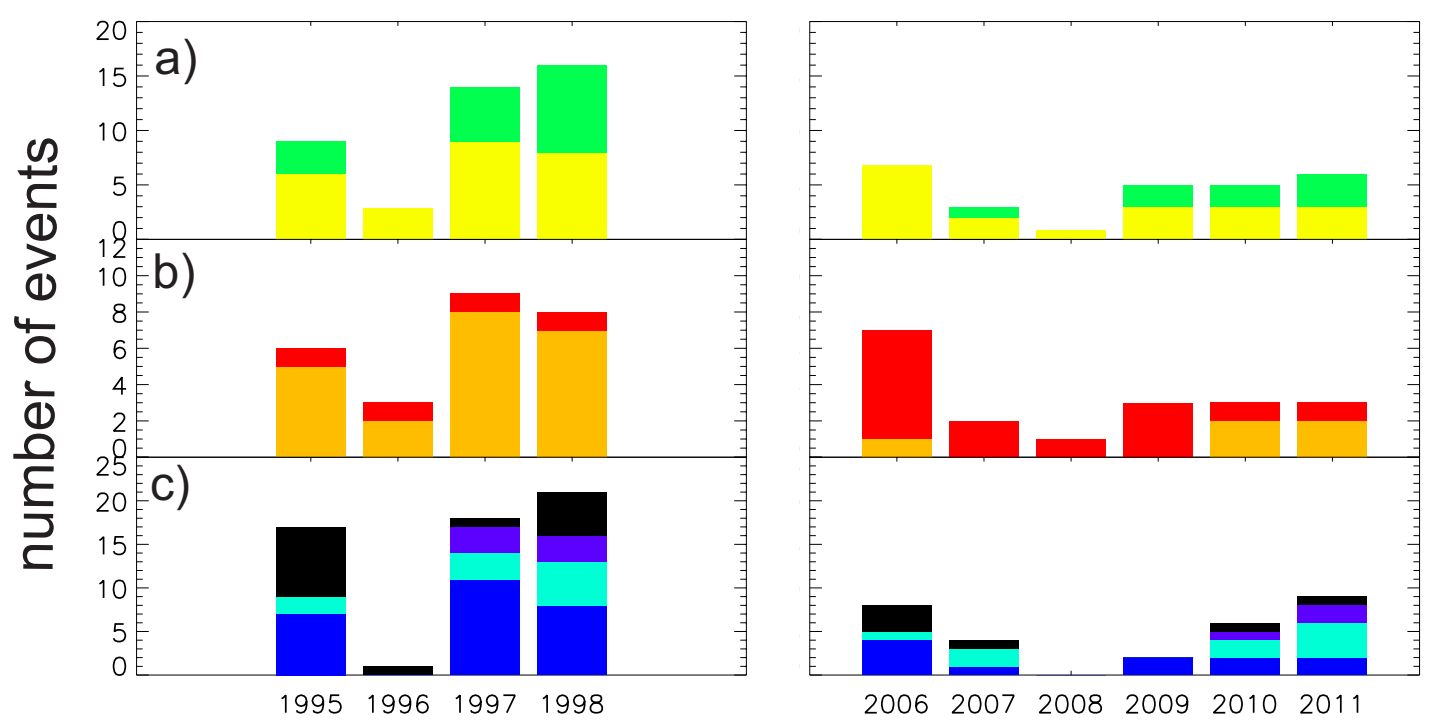

Fig. 3. Stacked histograms show annual counts of (a) bipolar (yellow bars) and unipolar (green bars) clouds, (b) original-SN (orange bars) and original-NS (red bars) clouds, (c) Dst $<-50 \mathrm{nT}$ magnetic storms as predicted from Eqs. (1)-(2) using measured $B_{\mathrm{Z}}$. In the bottom panel blue bars represent storms associated with magnetic clouds, light blue bars complex ICMEs, purple bars sheath regions and finally black bars give the number of storms related to non-CME solar wind.

magnetic field and density enhancements was also higher during Period 2.

\section{Statistical results}

\subsection{Annual counts of magnetic clouds and magnetic storms}

In total, our selection includes 69 magnetic clouds from which 42 were observed during Period 1 and 27 during Period 2. Panel (a) in Fig. 3 gives the yearly number of bipolar and unipolar magnetic clouds. During Period 1, the number of magnetic clouds decreased from nine events in 1995 to only three clouds in the solar minimum year 1996. In the early rising phase of cycle 23 , the magnetic cloud rate increased clearly: in 1997 and 1998, 14 and 16 clouds were observed, respectively. During Period 2 the number of clouds was relatively low. In 2006, seven clouds were reported, but in 2007 the number of clouds had already dropped to three and in 2008, only one cloud was reported. It is seen that despite increased solar activity in 2010-2011, the magnetic cloud rate remained rather low.

The yellow bars in Fig. 3a represent bipolar magnetic clouds, and the green bars unipolar magnetic clouds. For both investigated periods, bipolar clouds prevailed, but the percentage of unipolar clouds was somewhat higher during Period 1: in 1995-1998, $38 \%$ of magnetic clouds were unipolar while in 2006-2011, $29 \%$ were unipolar. It is seen that the number of unipolar clouds was highest during the ascending phase of solar activity.
In Fig. $3 b$ the orange bars give the number of original-SN clouds and the red bars the number of original-NS clouds. During Period 1, $85 \%$ of bipolar clouds were SN-clouds, while during Period 2, $74 \%$ of bipolar clouds were NSclouds. SN-clouds dominated throughout Period 1; in fact, only one opposite polarity cloud was detected each year. During the first four years (2006-2011) of Period 2 only one SNcloud was identified in total, but during the last two years (2010-2011) SN-clouds outnumbered NS ones four to two.

The bottom panels of Fig. 3c show the annual counts of Dst $<-50 \mathrm{nT}$ storms. Using the modified Burton's formula (Eqs. 1-2), we calculated Dst values using original $B_{\mathrm{Z}}$ for the whole Period 1 and Period 2. As mentioned in Sect. 2, we used estimated Dst instead of the measured Dst, because final Dst values were not yet available for all investigated years. Then, we surveyed solar wind plasma and magnetic field measurements as well as the ICME catalogues listed in Sect. 2 to identify drivers for all Dst $<-50$ storms. The blue bars give the number of storms caused by magnetic clouds; light blue bars by sheath regions, purple bars by complex ICMEs, and the black bars represent the non-CME-related storms (mainly caused by slow-fast stream interaction regions). Note that the storms driven by magnetic clouds and complex ICMEs also include storms, where sheath fields have given a contribution to the Dst excursions. Sheathassociated storms are those where the peak of the Dst was caused solely by the sheath region.

It is evident that considerably more Dst storms were observed during Period 1 than during Period 2. For example, in 1995, 17 storms were counted, while in 2006 only eight storms took place. During the early rising phase of cycle 23 
Table 4. The second (third) row gives the number of bipolar clouds that produced a stronger Dst response as SN-polarity (NS-polarity), as well as the percentages of the cases that were associated with a leading shock, increased trailing solar wind speed (HSS) and trailing density and magnetic field enhancements. For definitions, see Sect. 2.

\begin{tabular}{cccccc}
\hline Polarity & Number & Shock & HSS & $N_{\mathrm{TE}} / N_{\mathrm{LE}}>1.5$ & $B_{\mathrm{TE}} / B_{\mathrm{LE}}>1.1$ \\
\hline SN & 21 & $40 \%$ & $19 \%$ & $19 \%$ & $10 \%$ \\
NS & 20 & $52 \%$ & $70 \%$ & $60 \%$ & $75 \%$ \\
\hline
\end{tabular}

Table 5. The number of events when magnetic clouds of different $B_{\mathrm{Z}}$-types led to a Dst $<-50 \mathrm{nT}$ storm. The second column gives the total number of storms for Period 1 and Period 2 combined, and the next column the average Dst minimum. The last two columns give the number of storms during our two study periods separately.

\begin{tabular}{lcccc}
\hline Polarity & Total & Dst min (nT) & Period 1 & Period 2 \\
\hline original-SN & 17 & -81.4 & 15 & 2 \\
reversed-NS & 8 & -66.1 & 3 & 5 \\
original-NS & 9 & -66.3 & 1 & 8 \\
reversed-SN & 14 & -92.9 & 10 & 4 \\
original-S & 11 & -94.1 & 10 & 1 \\
reversed-N & 12 & -90.8 & 6 & 6 \\
original-N & 0 & - & 0 & 0 \\
reversed-S & 0 & - & 0 & 0 \\
\hline
\end{tabular}

(1997-1998), 39 storms occurred in total, while during the rising phase of the next cycle (2010-2011) only 15 storms were counted. By comparing the number of storms in each driver category between our two study periods, we see from Fig. $3 c$ that it is both the lack of non-CME storms and CMErelated storms that contributed to low geomagnetic activity during Period 2.

\subsection{Geoeffects from bipolar magnetic clouds}

The fast stream that was overtaking the magnetic cloud presented in Fig. 2 presumably increased the geoeffectiveness of the reversed-SN cloud. High densities in the cloud's tail part were related to filament material (Burlaga et al., 1998; Wu et al., 2000) that was further compressed by the following stream. The magnetic cloud was associated with a leading shock that arrived at the magnetopause on 10 January 1997 at 00:50 UT. The cloud's sheath region lasted only about $4 \mathrm{~h}$ and featured relatively low magnetic fields. Thus, sheath fields did not produce any significant geomagnetic consequences.

To further examine the geoeffectiveness of SN- versus NSpolarity (see Discussion in Sect. 1), we first tested for all 45 bipolar magnetic clouds whether they were more geoeffective with original or reversed polarity. The results are given in Table 4. In 20 cases, NS-polarity produced a stronger response in Dst, while in 21 cases SN-polarity led to a larger Dst excursion. From those clouds for which NS-polarity was more geoeffective $70 \%$ were trailed by higher-speed solar wind speed, and $60 \%$ and $75 \%$ were associated with trailing density and magnetic field enhancements. The corresponding percentages were clearly lower for clouds that were more geoeffective as SN-polarity, 19\%, $19 \%$ and $10 \%$, respectively. Table 4 also shows that the fraction of events associated to a leading shock is slightly higher for the clouds that produced a stronger storm as NS-polarity.

Table 5 gives the total number of Dst $<-50 \mathrm{nT}$ storms caused by different magnetic cloud types for our study periods. We have indicated separately the storms associated with the original and reverse cases. There were in total 27 original-SN clouds and 18 original-NS clouds. Thus, there are fewer storms associated with original-NS and reversedNS clouds than with original-SN and reversed-SN clouds. The storms driven by original-NS and reversed-NS clouds also produced weaker Dst storms. This is presumable due to the fact that the majority of original-NS clouds were identified during Period 2 when magnetic clouds were generally weaker than during Period 1; the average peak magnetic field magnitude of magnetic clouds in Period 1, was $16.5 \mathrm{nT}$, while during Period 2, $14.3 \mathrm{nT}$. When we add together the original-NS and reversed-SN clouds as well as the original-SN and reversed-NS clouds we see that the SN and NS-polarities were almost equally geoeffective: SN-polarity clouds caused $25 \mathrm{Dst}<-50 \mathrm{nT}$ storms with the average Dst minimum of $-79 \mathrm{nT}$, while the number of Dst storms related to NS-polarity clouds was 23 with the average Dst minimum of $-84 \mathrm{nT}$.

In Table 6, we have showed how a leading shock and a trailing solar wind speed structure affect the geoeffectivity of SN- and NS-polarity cases. Note that here we have grouped together original-NS and reversed-SN clouds as well as original-SN and reversed-NS clouds. It is seen that SNpolarity clouds produced Dst $<-50 \mathrm{nT}$ storms almost with the same frequency regardless of whether they had a leading shock or not. However, it is seen that the majority $(70 \%)$ of non-geoeffective $\mathrm{SN}$-polarity clouds did not drive a shock. It is also interesting to note that the association with a leading shock between geoeffective and non-geoeffective cases is rather similar for NS- and SN-polarity cases. $57 \%$ of geoeffective NS-polarity clouds were associated with a trailing higher-speed solar wind, while this was the case only for $32 \%$ of geoeffective SN-polarity clouds. In particular, the 


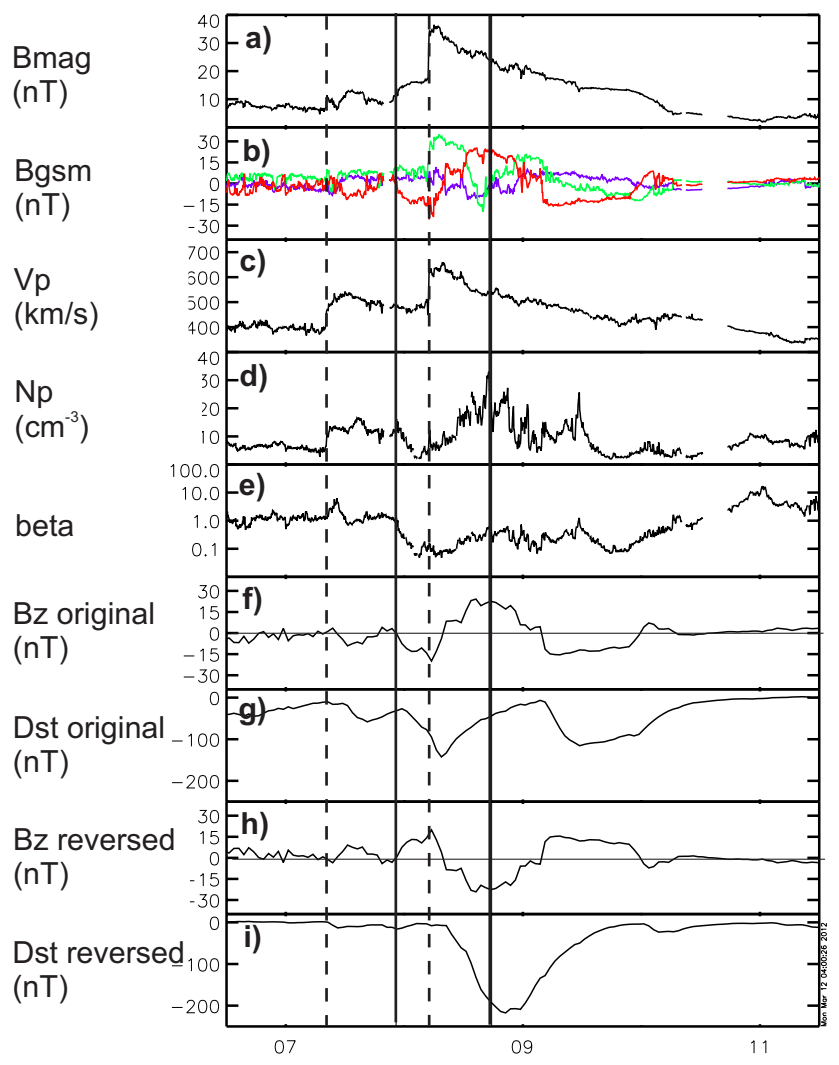

Fig. 4. Solar wind plasma and magnetic field measurements upstream of the Earth on 7-9 November 1998 featuring two interacting magnetic clouds. The storm driven by the reversed-SN cloud was the strongest in our dataset. The panels are same as in Fig. 2. The first dashed line shows the shock associated with the first cloud and the second dashed line indicates the shock driven by the following cloud.

majority of non-geoeffective NS-polarity clouds $(77 \%$ of events), but the majority (68\%) of geoeffective SN-polarity clouds lacked the trailing higher-speed stream.

Bipolar clouds (with original or reversed polarity) caused, in total, nine intense (Dst $<-100 \mathrm{nT}$ ) storms. Four of these storms were driven by shock-driving SN-polarity clouds, two by NS-polarity clouds that were associated with a higherspeed trailing solar wind and two by NS-polarity clouds overtaken by another ICME (see below). The remaining intense storm (13-14 April 2006) was driven by an NS-polarity cloud that was surrounded by a slow solar wind. This cloud had a magnetic field maximum of almost $20 \mathrm{nT}$ and although this peak value occurred approximately at the centre of the cloud where $B_{Z}$ was zero, the relatively high magnetic fields (about $15 \mathrm{nT}$ ) were detected at the cloud's boundaries.

The strongest storm caused by a bipolar cloud in our dataset took place in 7-8 November 1998. The solar wind magnetic field and plasma measurements as well as estimated Dst values are shown in Fig. 4. As shown by the solar wind plasma and magnetic field measurements an original-
Table 6. Distribution of geoeffective and non-geoeffective SN, NS and S-polarity clouds to cases that were related to a shock and a higher-speed trailing stream (HSS) and those that were not. The first number for each type indicates geoeffective cases and the second number non-geoeffective cases. Note that in this table we have grouped together original and reversed types. For example, column "SN" includes original-SN and reversed-NS clouds.

\begin{tabular}{lccc}
\hline & SN-polarity & NS-polarity & S-polarity \\
\hline Shock & $12 / 6$ & $14 / 4$ & $14 / 0$ \\
No shock & $13 / 14$ & $11 / 16$ & $10 / 1$ \\
HSS & $8 / 10$ & $13 / 5$ & $8 / 0$ \\
No HSS & $17 / 10$ & $10 / 17$ & $16 / 1$ \\
\hline
\end{tabular}

SN cloud was interacting with a unipolar (original polarity S) magnetic cloud. The first dashed line shows the shock associated with the bipolar cloud, while the second dashed line indicates the shock that was driven by the unipolar cloud. It is seen that this second shock was running into the preceding cloud. With the measured $B_{\mathrm{Z}}$ two intense Dst storms ensued: the first storm had Dst peak $-143 \mathrm{nT}$ caused by the southward leading fields of the original-SN cloud and the latter storm (peak $-119 \mathrm{nT}$ ) was related to southward fields of the S-type cloud. When we reversed the sign of $B_{\mathrm{Z}}$, the first magnetic cloud changed to reversed-SN cloud and the overtaking cloud compressed the southward fields and density in its end portion. These conditions led to a major storm with Dst minimum $-217 \mathrm{nT}$ reached on 8 November at 20:00 UT. With reversed $B_{\mathrm{Z}}$ the latter magnetic cloud had northward fields and Dst recovered as the cloud passed by the Earth. Our dataset includes also another event where the shock of the overtaking ICME propagated into a preceding bipolar cloud. This event was observed on 24-25 June 1998. The preceding cloud was an original-SN cloud and with this original polarity the Dst index did not cross our storm limit. However, when we changed the sign of $B_{\mathrm{Z}}$ the resulting reversed-SN cloud with its trailing fields compressed produced an intense Dst storm (minimum $-112 \mathrm{nT}$ ).

For the event shown in Fig. 2, the storm peaked $17 \mathrm{~h}$ earlier for the original-SN case than for the reversed-SN case. On average, for those $25 \mathrm{SN}$-polarity clouds that produced a Dst $<-50 \mathrm{nT}$ storm, the Dst minimum occurred $7.3 \mathrm{~h}$ after the cloud's leading edge had reached the magnetopause. For geoeffective NS-polarity clouds, the Dst peak was reached on average $20.2 \mathrm{~h}$ after the cloud's leading edge had arrived to the magnetopause. When a bipolar cloud caused a storm both with original and reversed polarity, the longest time difference we observed between the peaks of the storms was $21 \mathrm{~h}$ (for the 18-19 October 1995 magnetic cloud).

Our examination of the cloud's geoeffectiveness with the original and reversed polarity revealed a clear difference in the geoeffectiveness of opposite polarity clouds between our study periods: during Period 1 , the clouds were more 


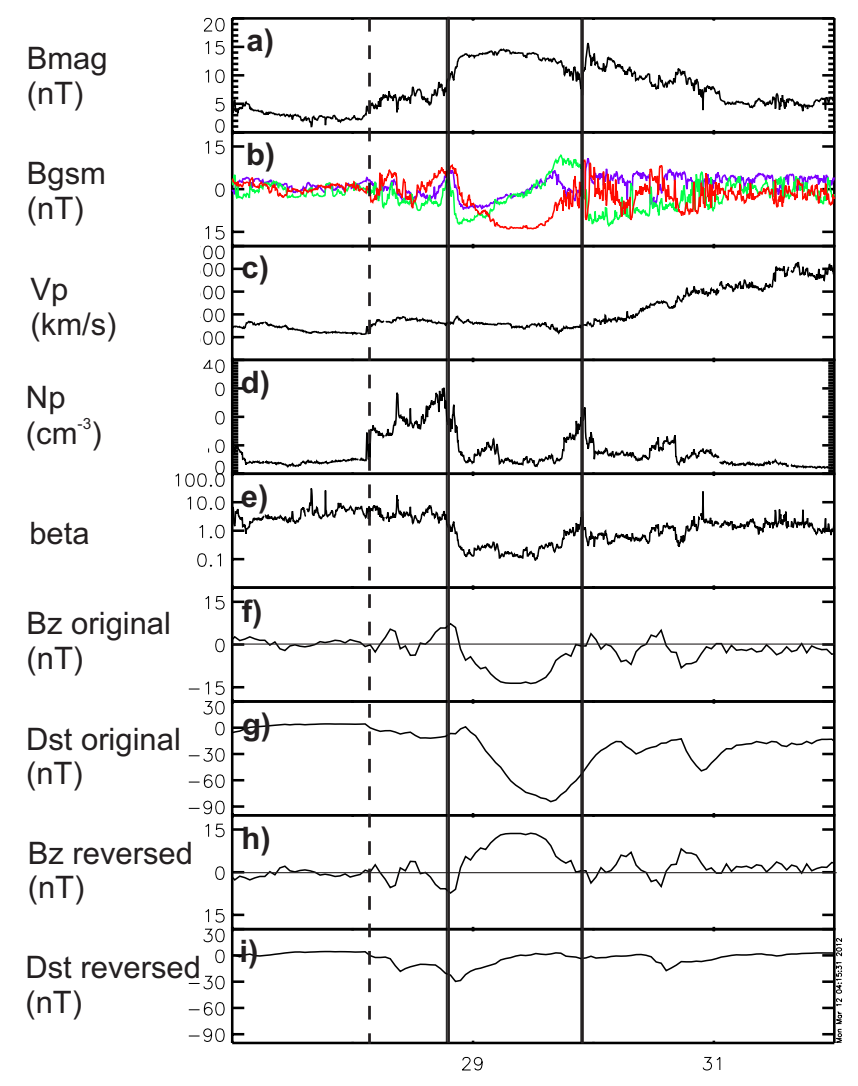

Fig. 5. Example of an unipolar magnetic cloud detected on 2829 May 2010. The panels are same as in Fig. 2.

geoeffective with SN-polarity in $65 \%$ of cases while during Period 2, the clouds caused a stronger Dst response with NS-polarity in $67 \%$ of cases. The different geoeffectivity of $\mathrm{SN}$ and NS-polarity cases between our study periods is also seen in Table 5, where the last two columns give the total number of Dst $<-50 \mathrm{nT}$ storms separately for Periods 1 and 2. During Period 1, there were 18 storms from SN-polarity clouds, and 11 storms from NS-polarity, while during Period 2, storms related to NS-polarity clouds outnumbered the storms driven by SN-polarity clouds by 12 to 7 .

\subsection{Geoeffects from unipolar magnetic clouds}

Approximately one-third of all magnetic clouds included in this study have unipolar $B_{\mathrm{Z}}$ behaviour. Figure 5 shows an original-S type cloud that was observed on 28-29 May 2010. The cloud drove a shock that arrived on the magnetopause on 28 May at 02:45 UT. The magnetic field magnitude in the sheath region was relatively low and the sheath did not cause a significant decrease in Dst. At the cloud's leading edge, the magnetic field started to turn southward and approximately at the middle of the cloud, $B_{\mathrm{Z}}$ had its maximum southward value. The Dst minimum $-84 \mathrm{nT}$ was reached on 29 May at 15:00 UT. When the sign of $B_{Z}$ was reversed, during the whole cloud's passage, the magnetic field pointed north and no Dst storm followed.

All S-polarity clouds (i.e., original-S or reversed-N cloud) in our dataset except one caused at least a moderate Dst storm, while for N-polarity clouds (original-N or reversed-S) no significant response in Dst ensued, see Table 5 (note that here we excluded cases where sheath fields alone drove a storm). In total S-polarity clouds caused 23 Dst storms with Dst minimum average of $-93 \mathrm{nT}$. The only unipolar cloud that did not cause a storm as S-polarity case was detected in 7-8 February 2010. This cloud did not have a leading shock and was embedded within a slow solar wind. The maximum magnetic field magnitude of this cloud was also relatively low, $10.7 \mathrm{nT}$ (i.e., just exceeding the threshold value we required for a magnetic cloud).

Table 6 shows that $58 \%$ of geoeffective S-polarity clouds were associated with a leading shock, while only about onethird was trailed by a faster solar wind stream. S-polarity clouds caused nine intense storms. Five of the clouds associated with intense Dst activity were shock-related, and three were followed by a higher-speed stream.

We found a clear difference in the number of original-S clouds between two investigated periods. During Period 1, ten original-S clouds were observed, while during Period 2, only one original-S cloud was detected. The number of original-N clouds was more similar between our study period: during four years of observations of Period 1, six original-N clouds were reported and during six years of observations of Period 2, seven original-N clouds were detected. Six of the nine original-S clouds counted for Period 1 occurred in 1998, and these clouds made a significant contribution to geomagnetic activity on that year. As seen from Fig. 3, 21 Dst $<-50 \mathrm{nT}$ storms took place in 1998 with six (29\%) of these storms caused by original-S clouds. Comparatively, in 2011, during approximately the same phase of the solar cycle and similar sunspot levels, only eight Dst storms occurred. Two of these storms were related to bipolar magnetic clouds and none to an original-S cloud.

\section{Discussion}

During Period 1, original-SN clouds clearly dominated original-NS clouds, consistent with the solar cycle rule for bipolar magnetic clouds (see Sect. 1). According to this rule, original-NS clouds should have prevailed throughout Period 2. This was the case during the late declining phase of cycle 23 and the following minimum, but during the rising phase of cycle 24 (years 2010-2011) more original-SN clouds were identified.

Because the annual counts of magnetic clouds were relatively low during Period 2, we checked the magnetic cloud rate from Solar TErrestrial RElations Observatory (STEREO; Kaiser et al., 2007). STEREO was launched in October 2006 and its two spacecraft have since separated 
from each other at the rate of $45^{\circ}$ per year. When STEREO magnetic clouds were added to our near-Earth events, the fraction of original-NS clouds increased to $60 \%$ in 2010 2011. Thus, our improved statistics show that original-NS clouds dominated also during the last two years of Period 2, but more "new polarity" clouds were identified than for the approximately same phase of the previous cycle. As discussed in the introduction for three previous solar cycles, one polarity clearly dominated during the whole rising phase and solar maximum and two polarities co-existed only during the declining phase. The relationship between the magnetic structure of interplanetary magnetic clouds and their solar source regions is complex (Li et al., 2011), and more detailed studies are needed to understand the solar cycle variations of bipolar magnetic clouds.

Our comparison of geomagnetic response of magnetic clouds with both original and reversed polarity showed that their geoeffectiveness depends evidently on the combination of the cloud's polarity and the ambient solar wind structure. We found that magnetic clouds overtaken by faster solar wind were associated more frequently with the tail part magnetic field and density enhancements than the clouds embedded in the slow or declining fast solar wind. The bipolar clouds that were associated with such a tail part compression caused evidently stronger storms as NS- than SN-polarity. In particular, bipolar clouds that lacked the trailing high-speed stream did not cause significant geomagnetic activity as NSpolarity, but were likely to drive a storm as SN-polarity. When a cloud is not compressed by a faster stream, it has typically experienced a significant expansion during its travel from the Sun to $1 \mathrm{AU}$, shifting the magnetic field maximum towards the leading edge (Gosling, 1990). Stronger leading magnetic fields increase geoeffectivity of SN-polarity clouds and this might explain why the majority of geoeffective SNpolarity clouds in our study occurred in slow or declining solar wind stream.

In addition, four of the five clouds caused intense Dst storms as NS-polarity were associated with a higher-speed solar wind stream. However, if an NS-polarity cloud is strong enough it can cause intense Dst activity even when surrounded by slow solar wind. Particularly strong storms may arise when an NS-polarity cloud is followed by another ICME. As shown by Lugaz et al. (2005) if the latter ICME drives a shock it will strengthen when it travels through a preceding low-beta region. This was apparently the case for the 7-8 November 1998 event where a shock of a trailing ICME travelled into a preceding cloud and the consequent magnetic field and density compressions led to the largest storm in our dataset. With the opposite polarity case (SNpolarity), the storm was considerably weaker (the Dst minima of the storm were $-217 \mathrm{nT}$ and $-143 \mathrm{nT}$ for the NSand $\mathrm{SN}$-polarity cases, respectively).

The question of how much pre-existing ring current contributes to incoming intensifications is particularly relevant for SN-polarity clouds. For NS-polarity clouds, sheath fields should not play an important role in increasing the geoeffectiveness of the cloud itself, because if the sheath drives geomagnetic activity it usually recovers to the quiet time level before the southward cloud fields arrive.

A simulation analysis of a three-peak magnetic storm by Kozyra et al. (2002) demonstrated that the final strength of the storm was not affected much when two previous intensifications were removed. The authors proposed that an enhanced convection electric field placed ring current particles to open drift trajectories and these particles were quickly removed from the magnetosphere. We found that a leading shock was not required for an SN-polarity cloud to drive a moderate storm, as about half our geoeffective SNpolarity clouds lacked a shock. However, the majority of nongeoeffective SN-polarity clouds lacked a shock, and all SNpolarity clouds that caused an intense storm drove a shock. Interestingly, we found that the dependence of geoeffectivity of NS-polarity clouds on the leading shock was rather similar to that of SN-polarity clouds. This may be attributed to the fact that shock-associated magnetic clouds tend to have stronger magnetic fields and, thus, lead to stronger storms. For our dataset, the average peak magnetic field magnitude for shock-driving clouds was $18.8 \mathrm{nT}$ and for non-shock driving clouds, $15.2 \mathrm{nT}$. Other studies have also demonstrated that faster magnetic clouds tend to have stronger peak magnetic field magnitudes (e.g. Gonzalez et al., 1998)

One important process that may enhance geoeffectivity of NS-polarity clouds is the magnetospheric preconditioning under northward IMF. Several studies (e.g., Borovsky et al., 1997; Jordanova et al., 1997; Lavraud et al., 1998) have shown that during the periods of extended northward IMF, a cold and dense plasma sheet is formed in the tail of the magnetosphere, and as a consequence, solar wind structures preceded by a northward IMF may be more geoeffective. As southward fields in NS-polarity clouds are preceded by a relatively long and intense northward IMF, it is expected that these conditions favour the formation of the cold and dense plasma sheet and, thus, may lead to a stronger ring current. Thus, to fully understand differences in the geoeffectivity between opposite polarity bipolar clouds requires a detailed understanding of solar wind-magnetospheric coupling processes and magnetospheric dynamics.

The polarity of bipolar clouds also affects the temporal evolution of a storm. As southward fields occur towards the end part of the cloud, the storm from NS-polarity clouds tend to occur significantly later than from SN-polarity clouds. We found that the peak of the storm from NS-polarity clouds occurred, on average, about half a day later than from SNpolarity clouds, but the difference can be almost one day. This should be noted when making space weather forecasts.

We found that S-polarity clouds were particularly geoeffective. Only one unipolar cloud in our dataset did not cause a Dst storm (not with the original nor with the reversed polarity), and the storms caused by S-polarity clouds were, on average, stronger than those from bipolar clouds. Only about 
one-third of geoeffective S-polarity clouds were associated with a faster solar wind stream. It is expected that the geoeffectivity of S-polarity clouds is not particularly sensitive for the surrounding solar wind structure as the storm is caused by the axial field that peaks at the center of the cloud.

The observed dependence of geoeffectivity of bipolar clouds on the ambient solar wind structure led to interesting differences between our two study periods. During Period 1, SN-polarity cases were clearly more geoeffective, while during Period 2, NS-polarity cases produced more storms. This discrepancy is presumably attributed to different large-scale solar wind structure during the investigated periods. As discussed in the introduction, the late declining phase of cycle 23 and the following solar minimum were featured by long-lived and low-latitude coronal holes resulting in frequent slow-fast stream interaction regions in the ecliptic plane. In addition, the ICMEs were generally slower during the recent solar minimum (Jian et al., 2011) so they were easier to be caught up with by the fast wind. As a consequence, as shown in Sect. 2, a considerably larger fraction of magnetic clouds during Period 2 were trailed by a higher-speed solar wind. This evidently increased the geoeffectivity of NSclouds during our latter study period.

Previous studies (Mulligan et al., 1998; Li and Luhmann, 2004; Huttunen et al., 2005) have not reported solar cycle dependence for the axial field orientation in unipolar clouds. However, we found a clear difference in the number of original-S clouds between our two study periods (during Period 1 ten original-S clouds were detected, while during Period 2 only one original-S cloud was observed). As discussed above, for unipolar clouds geomagnetic response depends drastically on the direction of the axial field. Thus, if the preferred axial field orientation changes between the cycles it might have significant consequences on geomagnetic activity. This was seen for our data when geomagnetic activity between years 1998 and 2011 were compared.

However, there is no obvious physical grounds to expect solar cycle variations for the axial field for unipolar clouds. Unipolar magnetic clouds may result from solar flux ropes that have originally high inclination, or they are intrinsically low-inclination ropes that have rotated after being released from the Sun. Several studies have indicated that left-handed flux ropes (i.e., clouds whose magnetic field rotates counterclockwise, types SEN, NWS) should rotate counterclockwise, and right-handed flux ropes (SWN and NES) should rotate clockwise (e.g., Green et al., 2007; Lynch et al., 2009). As a consequence, SWN and NES-clouds should produce high-inclination clouds with the axial field pointing north, and SEN and NWS-clouds with the axial field oriented south. Since the magnetic cloud handedness has no solar cycle dependence (because magnetic clouds come approximately with the same frequency from the Northern and Southern Hemispheres), the rotation of low-inclination clouds should produce equal amount of $\mathrm{S}$ - and $\mathrm{N}$-type clouds.
Because our statistics were relatively low, we also checked the number and types of unipolar magnetic clouds from STEREO for 2007-2011. In total, these two spacecraft identified five original-S clouds and seven original-N clouds. For Period $1,52 \%$ of unipolar clouds were original-S, while for Period 2, when unipolar clouds from STEREO were combined to near-Earth observations, the fraction is $33 \%$. This indicates a preference for northward axial direction in Period 2, but the difference is not so drastic as for the near-Earth observations only.

Our Period 2 was geomagnetically clearly quieter than Period 1. Our analysis shows that the low number of magnetic storms during Period 2 was attributed to the lack of both CME and non-CME associated storms. Several studies have shown that during the recent low solar activity period the near-ecliptic IMF and dynamic pressure were about $30 \%$ weaker (e.g., Lee et al., 2009; Jian et al., 2011), CMEs were slower (Vourlidas et al., 2011) and ICMEs (Kilpua et al., 2011; Kilpua et al., 2012; Jian et al., 2011) had weaker magnetic fields and lower speeds than reported during the previous solar minimum. This generally weaker IMF and dynamic pressure in stream interaction regions and in ICMEs presumably led to weaker geomagnetic consequences.

The overall distribution of storms between CME-driven (i.e., storms driven by magnetic clouds, complex ICMEs and sheath regions) and non-CME-associated storms was roughly similar between our two study periods. Except during the solar minimum years in 1996 and 2008, CMEs caused a majority of storms, in particular during the ascending solar activity phase. The increase in the number of sheath-driven storms during the rising phase of solar activity is presumably due to CMEs becoming faster when solar activity increases. However, the fraction of storms caused by complex ICMEs was slightly higher for Period 2, implying that ICMEs had generally more complex structure during our latter study period. This may be attributed to differences in CME initiation mechanisms itself, to the geometrical effect (i.e., how the spacecraft traverses the ICME) or to stronger deformation of ICMEs during this period.

\section{Conclusions}

In this paper, we have studied the geoeffectiveness of different types of magnetic clouds during two periods concentrated around the last two solar minima. We did not find an obvious difference in the total number and intensity of magnetospheric storms caused by bipolar clouds of the opposite polarity. However, our comparison of geomagnetic response of clouds with both original and reversed polarity show that during low and moderate solar activity, geoeffectivity of bipolar clouds differ significantly depending on the ambient solar wind conditions. Our two study periods showed clear differences in the solar wind structure surrounding the investigated magnetic clouds, and as a consequence, $\mathrm{SN}$ - and NS-polarity 
clouds had a different geoeffectivity. In particular, our study demonstrated that clouds that are followed by a higher-speed solar wind stream tend to cause stronger geomagnetic activity as NS-polarity, while when followed by a slow solar wind $\mathrm{SN}$-polarity is clearly more geoeffective.

The low geomagnetic activity during the early rising phase of solar cycle 24 was partly attributed to the lack of unipolar magnetic clouds with the southward oriented axial field. Even with the added events from STEREO, N-type unipolar clouds clearly outnumbered S-type clouds during our latter study period, but it is not clear whether this is a coincidence or whether there is a solar cycle dependence for unipolar clouds. We found a mixture of SN- and NS-clouds already during the early rising phase of cycle 24 , although for three previous solar cycles one polarity has dominated until the start of the declining phase.

Due to recent unprecedented solar conditions during the space era, it will be particularly interesting to witness how the preferred structure of magnetic clouds evolves for the rest of solar cycle 24 and during the subsequent cycles. In addition, a more detailed knowledge on solar wind magnetosphere coupling processes and magnetospheric dynamics is evidently needed to understand the differences in geomagnetic consequences of bipolar magnetic clouds. However, our study demonstrated that depending on the characteristics of the global solar magnetic field and the large-scale solar wind structure where magnetic clouds are injected, the geoeffectivity of different polarity clouds may change between the solar cycles.

Acknowledgements. The OMNI data were obtained from the GSFC/SPDF OMNIWeb interface at http://omniweb.gsfc.nasa.gov. We thank R. P. Lin for the WIND 3-D-plasma data, K. W. Ogilvie for the WIND SWE data, N. Ness for the ACE MFI data, and D. J. McComas for the ACE SWE data. Academy of Finland project 1218152 and NASA's STEREO grant NAS5-03131 are thanked for financial support. Y. Li is under the support of NASA GI grant NNX08AJ04G and NSF CISM ATM-0120950.

Topical Editor R. Forsyth thanks K. Marubashi and another anonymous referee for their help in evaluating this paper.

\section{References}

Abramenko, V., Yurchyshyn, V., Linker, J., Mikic, Luhmann, J., and Lee, C. O.: Low latitude coronal holes at the minimum of the 23rd solar cycle, Astrophys. J., 712, 813-818, 2010.

Borovsky, J. E., Thomsen, M. F., and McComas, D. J.: The superdense plasma sheet: Plasmaspheric origin, solar wind origin, or ionospheric origin?, J. Geophys. Res., 102, 22089-22106, 1997.

Bothmer, V. and Schwenn, R.: The structure and origin of magnetic clouds in the solar wind, Ann. Geophys., 16, 1-24, doi:10.1007/s00585-997-0001-x, 1998.

Burlaga, L.: Magnetic clouds and force-free fields with constant alpha, J. Geophys. Res., 93, 7217-7224, 1988.
Burlaga, L., Sittler, E., Mariani, F., and Schwenn, R.: Magnetic loop behind an interplanetary shock: Voyager, Helios and IMP 8 observations, J. Geophys. Res., 86, 6673-6684, 1981.

Burlaga, L. F., Lepping, R. P., and Jones, J. A.: Global configuration of a magnetic cloud, in Physics of Magnetic Flux Ropes, Geophys. Monogr., 58, Washington, AGU, 373-377, 1990.

Burlaga, L., Fitzenreiter, R., Lepping, R., Ogilvie, K., Szabo, A., Lazarus, A., Steinberg, J., Gloeckler, G., Howard, R., Michels, D., Farrugia, C., Lin, R. P., and Larson, D. E.: A magnetic cloud containing prominence material - January 1997, J. Geophys. Res., 103, 277-285, 1998.

Burton, R. K., McPherron, R. L., and Russell, C. T.: An empirical relationship between interplanetary conditions and Dst, J. Geophys. Res., 80, 4204-4214, 1975.

Cremades, H., Mandrini, C. H., and Dasso, S.: Coronal transient events during two solar minima: Their solar source regions and interplanetary counterparts, Solar Phys., 274, 233-249, doi:10.1007/s11207-011-9769-7, 2011.

Fenrich, F. R. and Luhmann, J. G.: Geomagnetic response to magnetic clouds of different polarity, Geophys. Res. Lett., 25, 29993002, 1998.

Goldstein, H.: On the field configuration in magnetic clouds, in: Solar Wind Five, edited by: Neugebauer, M., Geophys. NASA Conf. Publ., 731-733, 1983.

Gonzalez, W. D., Clua de Gonzalez, A. L., Dal Lago, A., Tsurutani, B. T., Arballo, J. K., Lakhina, G. K., Buti, B., Ho, C. M., and Wu, S.-T.: Magnetic cloud field intensities and solar wind velocities, Solar Phys., 25, 963-966, 1998.

Gosling, J. T.: Coronal mass ejections and magnetic flux ropes in interplanetary space, in: Physics of Magnetic Flux Ropes, Geophys. Monogr., 58, edited by: Priest, E. R., Lee, L. C., and Russell, C. T., 343-364, 1990.

Green, L. M., Kliem, B., Torok, T., van Driel-Gesztelyi, L., and Attrill, G. D. R.: Transient coronal sigmoids and rotating erupting flux ropes, Solar Phys, 246, 365-391, doi:10.1007/s11207-0079061-z, 2007.

Huttunen, K. E. J., Schwenn, R., Bothmer, V., and Koskinen, H. E. J.: Properties and geoeffectiveness of magnetic clouds in the rising, maximum and early declining phases of solar cycle 23, Ann. Geophys., 23, 625-641, doi:10.5194/angeo-23-625-2005, 2005.

Jian, L. K., Russell, C. T., Luhmann, J. G., and Skoug, R. M.: Properties of interplanetary coronal mass ejections at one AU during 1995-2004, Solar Phys., 239, 393-436, 2006.

Jian, L. K., Russell, C. T., Luhmann, J. G., Skoug, R. M., and Steinberg, J. T.: Stream interactions and interplanetary coronal mass ejections at 0.72 AU, Solar Phys., 249, 85-101, doi:10.1007/s11207-008-9161-4, 2008.

Jian, L. K., Russell, C. T., and Luhmann, J. G.: Comparing solar minimum 23/24 with historical solar wind records at $1 \mathrm{AU}$, Solar Phys., 274, 321-344, doi:10.1007/s11207-011-9737-2, 2011.

Jordanova, V. K., Farrugia, C. J., Janoo, L., Quinn, J. M., Torbert, R. B., Ogilvie, K. W., Lepping, R. B., Steinberg, J. T., McComas, D. J., and Belian, R. D.: October 1995 magnetic cloud and accompanying storm activity: Ring current evolution, J. Geophys. Res., 103, 79-92, 1998.

Kaiser, M., Kucera, T. A., Davila, J. M., and St. Cyr, O. C.: Guhathakurta, M., and Christian, E: The STEREO mission: An introduction, Space Sci. Rev., 136, 5-16, doi:10.1007/s11214- 
007-9277-0, 2007.

Kilpua, E. K. J., Lee, C. O., Luhmann, J. G., and Li, Y.: Interplanetary coronal mass ejections in the near-Earth solar wind during the minimum periods following solar cycles 22 and 23, Ann. Geophys., 29, 1455-1467, doi:10.5194/angeo-29-14552011, 2011.

Kilpua, E. K. J., Jian, L. K., Li, Y., Luhmann, J. G., and Russell, C. T.: Observations of ICMEs and ICME-like solar wind structures from 2007-2010 using near-Earth and STEREO observations, Solar Phys., doi:10.1007/s11207-012-9957-0, in press, 2012.

Kozyra, J. U., Liehmohn, M. W., Clauer, C. R., Ridley, A. J., Thomsen, M. F., Borovsky, J. E., Roeder, J. L., Jordanova, V. K., and Gonzalez, W. D.: Multistep Dst development and ring current composition changes during the 4-6 June 1991 magnetic storm, J. Geophys. Res., 107, 1224, doi:10.1029/2001JA000023, 2002.

Lavraud, B., Thomsen, M. F., Borovsky, J. E., Denton, M. H., and Pulkkinen, T. I.: Magnetosphere preconditioning under northward IMF: Evidence from the study of coronal mass ejection and corotating interaction region geoeffectiveness, J. Geophys. Res., 111, A09208, doi:10.1029/2005JA011566, 2006.

Lee, C. O., Luhmann, J. G., Zhao, X. P., Liu, Y., Riley, P., Arge, C. N., Russell, C. T., and de Pater, I.: Effects of the weak polar fields of solar cycle 23: investigation using OMNI for the STEREO mission period, Solar Phys., 256, 345-363, doi:10.1007/s11207009-9345-6, 2009.

Li, Y. and Luhmann, J. G.: Solar cycle control of the magnetic cloud polarity and the geoeffectiveness, J. Atmos. Solar-Terr. Phys., 66, 323-331, 2004.

Li, Y., Luhmann, J. G., Lynch, B. J., and Kilpua, E. K. J.: Cyclic reversal of magnetic cloud poloidal field, Solar Phys., 270, 331346, doi:10.1007/s11207-011-9722-9, 2011.

Lugaz, N., Manchester, W. B., and Gombosi, T. I.: Numerical simulation of the interaction of two coronal mass ejections from Sun to Earth, Astrophys. J., 643, 651, doi:10.1086/491782, 2005.

Lundquist, S.: Magnetohydrostatic fields, Ark. Fys., 2, 361-365, 1950.

Lynch, B. J., Antiochos, S. K., Li, Y., Luhmann, J. G., and DeVore, C. R.: Rotation of coronal mass ejection during eruption, Astrophys. J., 697, 1918-1927, 2009.
Mulligan, T., Russell, C. T., and Luhmann, J. G.: Solar cycle evolution of the structure of magnetic clouds in the inner heliosphere, Geophys. Res. Lett., 25, 2959-2962, 1998.

Murayama, T.: Coupling function between solar wind parameters and geomagnetic indices, Rev. Geophys. Space Phys., 20, 623629, 1982.

O'Brien, T. P. and McPherron, R. L.: An empirical phase space analysis of ring current dynamics: Solar wind control of injection and decay, J. Geophys. Res., 105, 7707-7719, 2000.

Pulkkinen, T. I., Palmroth, M., Tanskanen, E. I., Ganushkina, N. U., Shukhtina, M. A., and Dmitrieva, N. P.: Solar windmagnetosphere coupling: A review of recent results, J. Atmos. Solar-Terr. Phys., 69, 256-264, 2007.

Richardson, I. G. and Cane, H. V.: The fraction of interplanetary coronal mass ejections that are magnetic clouds: Evidence for a solar cycle variation, Geophys. Res. Lett., 31, L18804, doi:10.1029/2004GL020958, 2004.

Richardson, I. G., Cliver, E. W., and Cane, H. V.: Sources of geomagnetic storms for solar minimum and maximum conditions during 1972-2000, J. Geophys. Res., 28, 2569-2572, 2001.

Russell, C. T., Luhmann, J. G., and Jian, J. K.: How unprecedented a solar minimum?, Rev. Geophys., 48, 16, doi:10.1029/2009RG000316, 2010.

Vourlidas, A., Howard, R. A., Esfandiari, E., Patsourakos, S., Yashiro, S., and Michalek, G.: Erratum: "Comprehensive analysis of coronal mass ejection mass and energy properties over a full solar cycle", Astrophys. J., 730, 1, 2011.

Zhang, G. and Burlaga, L. F.: Magnetic clouds, geomagnetic disturbances and cosmic ray decreases, J. Geophys. Res., 93, 25112518, 1988.

Webb, D. F., Cliver, E. W., Crooker, N. U., St. Cry, O. C., and Thomson, B. J.: Relationship of halo coronal mass ejections, magnetic clouds, and magnetic storms, J. Geophys. Res., 105 7491-7508, 2000.

Wu, Y. Q., Tang, Y. H., Dai, Y., and Wu, C. P.: The solar origin of the 6 January 1997 coronal mass ejection, Solar Phys., 207, 159-171, 2002. 